\title{
An energy-based framework for dense $3 D$ registration of volumetric brain images
}

\author{
Pierre Hellier Christian Barillot Étienne Mémin Patrick Pérez \\ IRISA, INRIA-CNRS, University of Rennes 1 \\ Campus de Beaulieu, F-35042 Rennes cedex, France. \\ http://www.irisa.fr/vista/
}

\begin{abstract}
In this paper we describe a new method for medical image registration. The registration is formulated as a minimization problem involving robust estimators. We propose an efficient hierarchical optimization framework which is both multiresolution and multigrid. An anatomical segmentation of the cortex is introduced in the adaptive partitioning of the volume on which the multigrid minimization is based. This allows to limit the estimation to the areas of interest, to accelerate the algorithm, and to refine the estimation in specified areas. Furthermore we introduce a methodology to constrain the registration with landmarks such as anatomical structures. The performances of this method are objectively evaluated on simulated data and its benefits are demonstrated on a large database of real acquisitions.
\end{abstract}

keywords Registration, atlas matching, medical imaging, local constraints, incremental optical flow, multigrid minimization, robust estimators.

\section{Introduction}

\subsection{Context}

Since a few years, the ways of observing human brain have tremendously evolved. Nowadays, surgeons must face not only the huge volume of data, but also the complementarity between the different images. As a matter of fact, the different acquisitions are not redundant but complementary, and should not be neglected for the patient's health. Medical image registration has thus become a crucial issue.

The non-linear registration of brains from different subjects allows to build an anatomical atlas of the cortex. Some atlases [15, 17] already exist, but they appear to be inadequate, because they often lack legibility and capacity to evolve, and their interpretation is very difficult. The major problem in building an atlas is the important variability of the human brain.
It has been clearly shown [15] that we cannot assume topological equivalence between two different brains. Considering the same sulcus of different subjects, one may find large differences of orientation, size, and even topology (one sulcus may be interrupted or absent for instance). "Voxel-based" approaches generally fail to take into account this variability, therefore we think it is necessary to constrain locally the registration process with relevant anatomical information.

\subsection{Related work}

Medical image registration is a very productive field, from a bibliographical point of view. A complete review and classification of different registration procedures is presented in [12]. Among them we have selected a few methods.

Because a major problem is the huge amount of data, some authors proposed methods to focus on peculiar structures to be extracted and matched. These structures may be points [3], curves [16], or surfaces [20]. The extraction of these landmarks is of course a critical issue, but the way these landmarks are matched -and the way the registration is then computed throughout the volume- is also critical. Methods have been developed to overcome this problem: the TPS algorithm [3], spline transformations, or the ICP algorithm [16].

Other registration methods are inspired by mechanical models, either elastic [1], or fluid [4]. Fluid models allow to reach, in theory, any displacements, but these methods are highly time-consuming. Christensen recently proposed [4] an interesting evolution of these methods, where the direct deformation field and the inverse deformation field are jointly estimated in order to guarantee the coherence of the deformation.

Finally, many non-rigid registration procedures are "voxel-based" methods: Thirion [19] proposes the demon method; Collins et al. [6] estimates a piecewise constant transformation that maximizes the cross cor- 
relation of the image gradient. Collins et al. recently proposed [7] to introduce cortical constraints in the registration process by computing a chamfer distance between selected sulci. Musse et al. [14] propose a method, which is much related to the method we introduce, based on the minimization of the displaced frame difference (DFD). This similarity measure is highly nonlinear and is not robust to the acquisition artifacts of MRI.

\subsection{Method}

The method proposed in this paper is an extension and a complete validation of our previous work presented in $[9,13]$. The registration problem is expressed as a motion estimation problem. Our $3 D$ method performs a non-rigid monomodality registration of MRI acquisition of different subjects. The similarity measure that we use incorporates robust estimators whose utility is twofold: on the one hand we want to limit the influence of the acquisition noise, on the other hand we want to be able to estimate transformations that modify the topology of the structures.

Many tasks in computer vision may be expressed as the minimization of a cost function. The optimization involves a very large number of variables, therefore efficient iterative multigrid approaches have been developed and applied in vision [8, 18].

To take into account large deformations, we use a multiresolution scheme. Besides, at each resolution level, we use a multigrid minimization to accelerate the algorithm and improve the quality of the estimation. Throughout this hierarchical approach, we designed an adaptive partition of the volume to refine the estimation on the regions of interest and avoid useless efforts elsewhere. An anatomical segmentation of the cortex is introduced and used in two ways: at each resolution level, we initialize the partition as an octree subdivision with the use of the segmentation, and the segmentation mask is used in the subdivision criterion to refine the estimation on the cortex.

Inside the multigrid minimization, we use a local parametric model for the deformation field and we introduce a formalism to constrain locally the registration process with relevant landmarks such as anatomical structures.

\section{Description of the registration method}

\subsection{General formulation}

The optical flow hypothesis, introduced by Horn et Schunck [10], assumes that the luminance of a physical point does not vary much between the two volumes to register. It gives: $f\left(s+\boldsymbol{w}_{s}, t_{1}\right) \Leftrightarrow f\left(s, t_{2}\right)=0$ where $s$ is a voxel of the volume, $t_{1}$ and $t_{2}$ are the indexes of the volumes (temporal indexes for a dynamic acquisition, indexes in a database for multi-subject registration), $f$ is the luminance function and $\boldsymbol{w}$ the expected $3 D$ displacement field. Generally, a linear expansion of this equation is preferred : $\boldsymbol{\nabla} f(s, t) \cdot \boldsymbol{w}_{s}+f_{t}(s, t)=0$ where $\nabla f(s, t)$ stands for the spatial gradient of luminance and $f_{t}(s, t)$ is the voxelwise difference between the two volumes. The registration problem may be formulated as the minimization of the following cost function:

$$
\begin{array}{r}
U(\boldsymbol{w} ; f)=\sum_{s \in S}\left[\boldsymbol{\nabla} f(s, t) \cdot \boldsymbol{w}_{s}+f_{t}(s, t)\right]^{2} \\
+\alpha \sum_{<s, r>\in \mathcal{C}}\left\|\boldsymbol{w}_{s}-\boldsymbol{w}_{r}\right\|^{2}
\end{array}
$$

where $S$ is the voxel lattice, $\mathcal{C}$ is the set of neighboring pairs (the 6 -neighborhood system may be used for instance) and $\alpha$ controls the balance between the two energy terms. The first term represents the interaction between the field (unknown variables) and the data (given variables), whereas the second term expresses the smoothness constraint. The weaknesses of this formulation are known: [a.] The optical flow constraint (OFC) is not valid in case of large displacements because of the linearization. [b.] The OFC might not be valid in all the regions of the volume, because of the noise of acquisition, intensity non-uniformity in MRI data, and occlusions. [c.] The "real" field is not globally smooth and it probably contains discontinuities that might not be preserved because of the quadratic cost.

To cope with the (b) and (c) limitations, we replace the quadratic cost by robust functions. To face the problem (a), we use a multiresolution scheme and a multigrid strategy to improve the minimization at each resolution level.

\subsection{Robust estimators}

Cost function (1) does not make any difference between relevant data and inconsistent data, nor between neighboring pairs where the field is smooth and neighboring pairs where the field is discontinuous. Therefore, we introduce robust functions and more precisely robust M-estimators [2]. An M-estimator is a function $\rho$ that is increasing on $\mathbb{R}^{+}$, such that (ii) $\phi(u) \triangleq \rho(\sqrt{u})$ is strictly concave on $\mathbb{R}^{+}$and (iii) $\lim _{x \rightarrow \infty} \rho^{\prime}(x)<\infty$.

(i) implies that $\rho$ is a cost function. (ii) implies that the graph of $\rho$ is the inferior envelope of a set of parabolas. We have:

$\exists \psi \in C^{1}([0, M], \mathbb{R}): \forall u, \rho(u)=\min _{z \in[0, M]}\left(z u^{2}+\psi(z)\right)$, 
where $M \triangleq \lim _{u \rightarrow 0^{+}} \phi^{\prime}(u)$. Furthermore one gets:

$$
z^{*} \triangleq \arg \min _{z \in[0, M]}\left(z u^{2}+\psi(z)\right)=\frac{\rho^{\prime}(u)}{2 u}=\phi^{\prime}\left(u^{2}\right),
$$

where $\frac{\rho^{\prime}(u)}{2 u}=\phi^{\prime}\left(u^{2}\right)$ decreases from $M$ to 0 according to (ii) and (iii).

The robustness of such an estimator is provided by the fact that the function $\phi^{\prime}$ decreases. We introduce two robust estimators, the first one on the data term $\left(\rho_{1}\right)$ and the second one on the regularization term $\left(\rho_{2}\right)$.

According to (2), the minimization of the cost function $U$ in (1) is equivalent to the minimization of the augmented function, noted $\stackrel{\star}{U}$ :

$$
\begin{gathered}
\stackrel{\star}{U}(\boldsymbol{w}, \delta, \beta ; f)=\sum_{s \in S} \delta_{s}\left(\boldsymbol{\nabla} f(s, t) \cdot \boldsymbol{w}_{s}+f_{t}(s, t)\right)^{2}+\psi_{1}\left(\delta_{s}\right) \\
+\alpha \sum_{<s, r>\in \mathcal{C}} \beta_{s r}\left(\left\|\boldsymbol{w}_{s}-\boldsymbol{w}_{r}\right\|\right)^{2}+\psi_{2}\left(\beta_{s r}\right)
\end{gathered}
$$

where $\delta_{s}$ and $\beta_{s r}$ are auxiliary variables (acting as "weights") to be estimated within $\left[0, M_{1}\right]$ and $\left[0, M_{2}\right]$ respectively. This cost function has the advantage to be quadratic with respect to $\boldsymbol{w}$.

\subsection{Multiresolution and multigrid}

In case of large displacements, we use a classical incremental multiresolution procedure (see fig. 1). We construct a pyramid of volumes $\left\{f^{k}\right\}$ by successive Gaussian smoothing and subsampling in each direction. At the coarsest level, displacements are reduced, and cost function (4) can be used. For the next resolution levels, only an increment $\mathrm{d} \boldsymbol{w}^{k}$ is estimated to refine estimate $\hat{\boldsymbol{w}}^{k}$, obtained from the previous level. This is done using cost function (1) but with $\nabla \tilde{f}_{s}^{k} \triangleq$ $\boldsymbol{\nabla} f^{k}\left(s+\hat{\boldsymbol{w}}_{s}^{k}, t_{2}\right)$ and $\tilde{f}_{t}^{k}(s, t) \triangleq f^{k}\left(s+\hat{\boldsymbol{w}}_{s}^{k}, t_{2}\right) \Leftrightarrow f^{k}\left(s, t_{1}\right)$ instead of $\boldsymbol{\nabla} f^{k}(s, t)$ and $f_{t}^{k}(s, t)$. As previously, this quantities come from the linearization of the constancy assumption expressed for the whole displacement $\hat{\boldsymbol{w}}_{s}^{k}+\mathrm{d} \boldsymbol{w}_{s}^{k}$. Also regularization term becomes $\sum_{<s, r>\in \mathcal{C}} \rho_{2}\left(\left\|\hat{\boldsymbol{w}}_{s}^{k}+\mathrm{d} \boldsymbol{w}_{s}^{k} \Leftrightarrow \hat{\boldsymbol{w}}_{r}^{k} \Leftrightarrow \mathrm{d} \boldsymbol{w}_{r}^{k}\right\|\right)$.

Furthermore, at each level of resolution, we use a multigrid minimization (see Fig. 2) based on successive partitions of the initial volume. At each grid level $\ell$, corresponding to a partition of cubes, we estimate a parametric increment field for each cube of the partition. The energy is consequently smoother, and has fewer local minima. The result is then used to initialize the next level. This minimization strategy, where the starting point is provided by the previous result which we hope to be a rough estimate of the desired solution -, improves the quality and the convergence

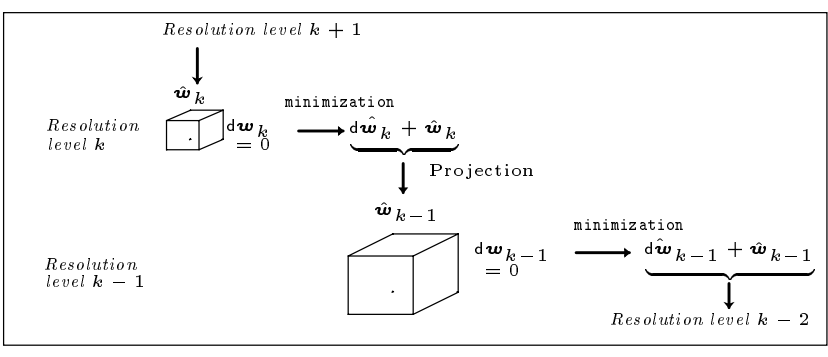

Figure 1: Incremental estimation of the optical flow.

rate as compared to standard iterative solvers (such as Gauss-Seidel).

To initialize the partition at the coarsest grid level $L$, we consider a segmentation of the cortex obtained by morphological operators. After a threshold and an erosion of the initial volume, a region growing process is performed from a starting point that is manually chosen. A dilatation operation allows us to end up with a binary segmentation. At grid level $L$, the partition is initialized by a single cube of the volume size. We iteratively divide each cube while it intersects the segmentation mask and while its size is superior to $2^{3 L}$. We finally get an octree partition which is anatomically relevant.

When we change of grid level, each cube is adaptively divided. The subdivision criterion depends first on the segmentation mask, but it also depends on the local distribution of the variables $\delta_{s}$ which reflects the local adequation between the data and the estimated deformation field.

\subsection{Parametric model}

We now introduce the deformation model that is used. We chose to consider an affine 12-parameter model on each cube of the partition.

At a given resolution level $k$ and grid level $\ell, \Xi_{k, \ell}=$ $\left\{\Xi_{n}, n=1 \cdots N_{k, \ell}\right\}$ is the partition of the volume into $N_{k, \ell}$ cubes $\Xi_{n}$. On each cube $\Xi_{n}$, we estimate an affine displacement increment defined by the parametric vector $\Theta_{n}^{\ell}: \forall s=(x, y, z) \in \Xi_{n}, \mathrm{~d} \boldsymbol{w}_{s}=P_{s} \Theta_{n}^{\ell}$, with $P_{s}=\mathbb{I}_{3} \otimes\left[\begin{array}{llll}1 & x_{s} & y_{s} & z_{s}\end{array}\right]$ (operator $\otimes$ denotes the Kronecker product).

A neighborhood system $V^{\ell}$ on the partition $\Xi_{k, \ell}$ derives naturally from the one, denoted $\mathcal{V}$, that equips $S$. It is defined as follows:

$\forall n, m \in\left\{1 \cdots N_{k, \ell}\right\}, m \in V^{\ell}(n) \Leftrightarrow \exists s \in \Xi_{n}, \exists r \in$ $\Xi_{m} / r \in \mathcal{V}(s) . \mathcal{C}$ being the set of neighboring pairs on $S^{k}$, we must now distinguish between two types of such pairs: the pairs inside one cube and the pairs between two cubes: 


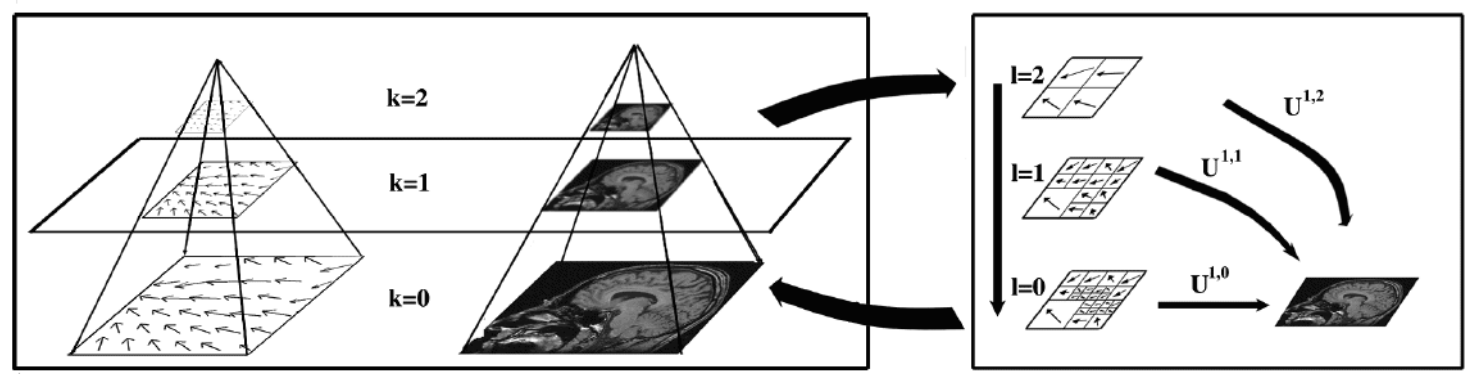

Figure 2: Example of multiresolution/multigrid minimization. For each resolution level (on the left), a multigrid strategy (on the right) is performed. For legibility reasons, the figure is a $2 D$ illustration of a $3 D$ algorithm with volumetric data. $\mathcal{V}(s)$.

$\forall n \in\left\{1 \ldots N_{k, \ell}\right\},<s, r>\in \mathcal{C}_{n}^{\ell} \Leftrightarrow s \in \Xi_{n}, r \in \Xi_{n}$ and $r \in$

$\forall n \in\left\{1 \ldots N_{k, \ell}\right\}, \forall m \in V^{\ell}(n),<s, r>\in \mathcal{C}_{n m}^{\ell} \Leftrightarrow m \in$ $V^{l}(n), s \in \Xi_{n}, r \in \Xi_{m}$ and $r \in \mathcal{V}(s)$.

For sake of concision, we will now drop the resolution index $k$. With these notations, the cost function (4) becomes:

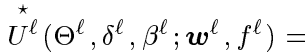

$\sum_{n=1}^{N_{\ell}} \sum_{s \in \Xi_{n}} \delta_{s}^{\ell}\left[\nabla \tilde{f}_{s}^{T} P_{s} \Theta_{n}^{\ell}+\tilde{f}_{t}(s, t)\right]^{2}+\psi_{1}\left(\delta_{s}^{\ell}\right)$

$+\frac{\alpha}{2} \sum_{n=1}^{N_{\ell}}\left[\sum_{m \in V^{\ell}(n)} \sum_{\left\langle s, r>\in \mathcal{C}_{n m}^{\ell}\right.} \beta_{s r}^{\ell}\left\|\left(\boldsymbol{w}_{s}^{\ell}+P_{s} \Theta_{n}^{\ell}\right) \Leftrightarrow\left(\boldsymbol{w}_{r}^{\ell}+P_{r} \Theta_{m}^{\ell}\right)\right\|^{2}\right.$ $\left.+\psi_{2}\left(\beta_{s r}^{\ell}\right)\right]$

$+\alpha \sum_{n=1}^{N_{\ell}}\left[\sum_{<s, r>\in \mathcal{C}_{n}^{\ell}} \beta_{s r}^{\ell}\left\|\left(\boldsymbol{w}_{s}^{\ell}+P_{s} \Theta_{n}^{\ell}\right) \Leftrightarrow\left(\boldsymbol{w}_{r}^{\ell}+P_{r} \Theta_{n}^{\ell}\right)\right\|^{2}\right.$

$$
\left.+\psi_{2}\left(\beta_{s r}^{\ell}\right)\right]
$$

Considering the auxiliary variables $\delta^{\ell}$ and $\beta^{\ell}$ as fixed, one can easily differentiate the cost function (5) with respect to $\Theta_{n}^{\ell}$ and get a linear system to be solved. In turn, when the deformation field is "frozen", the weights are obtained in a closed form from equation (3). The minimization may therefore be naturally handled in an alternated way.

\subsection{Cooperation with local constraints}

It may be interesting to constrain locally the registration process with landmarks. The registration of brains of different subjects is difficult due to the huge inter-subject variability, specially in the cortex area (see [7]). Therefore introducing local cortical constraints in the registration process would be an appealing way of apprehending inter-subject variability ([11]). Although we have not yet experienced such an extension, it can be easily expressed in our generic energy-based formalism as we now explain. Let us note $M$ the number of landmarks (cortical folds or important anatomical structures such as ventricles) that can be extracted for each subject. $\forall i \in[0, M], N_{i}$ is the number of points representing the landmark $i$. For landmark $i$, let us note $S_{1}^{i}=\left\{C_{i, j}^{S}, j \in\left[1, N_{i}\right]\right\}$ the set of points for the source volume and $S_{2}^{i}=\left\{C_{i, j}^{T}, j \in\right.$ $\left.\left[1, N_{i}\right]\right\}$ the set of corresponding points for the target volume. We compute a displacement vector:

$$
\forall i \in[1, M], \forall j \in\left[1, N_{i}\right], s=C_{i, j}^{S}, \boldsymbol{w}_{s}^{c}=C_{i, j}^{C_{i, j}^{T}}
$$

where $\boldsymbol{w}^{c}$ is the constraint deformation field. We note $\mathcal{S}_{c}=\left\{C_{i, j}^{S}, i \in[1, M], j \in\left[1, N_{i}\right]\right\}$ the support of the sparse deformation field.

The introduction of this constraint in the formulation of the problem is naturally performed by extending the objective function $\stackrel{\star}{U}$. Let us note $\breve{U}$ the new augmented objective function:

$\breve{U}\left(\mathrm{~d} \boldsymbol{w}, \delta, \beta ; \boldsymbol{w}^{c}, \boldsymbol{w}, f\right)=\stackrel{\star}{U}(\mathrm{~d} \boldsymbol{w}, \delta, \beta ; \boldsymbol{w}, f)+\alpha^{c} \sum_{s \in \mathcal{S}_{c}}\left\|\boldsymbol{w}_{s}+\mathrm{d} \boldsymbol{w}_{s} \Leftrightarrow \boldsymbol{w}_{s}^{c}\right\|^{2}$.

We could also introduce a robust estimator on the local constraint term. It depends on the type of landmark that is introduced and on the confidence about its extraction.

Note that the formulation is still quadratic w.r.t. the increment displacement model $\Theta^{\ell}$. The constraints appear in the cost function only for a finite number of voxels. Nevertheless, these local constraints will propagate through the cube on which we calculate the parametric increment, and they will also be propagated through the volume by the regularization term.

\section{Results}

\subsection{Experiments on simulated data}

To evaluate the registration method, we used the simulated data of the MNI (Brainweb : 
http://www.bic.mni.mcgill.ca/brainweb)

$[5]$. Data have been collected with 3 levels of noise and inhomogeneity. We designed a synthetic deformation field made up of a global affine field with large deformations combined with local stochastic perturbations. We did not try to build a "realistic" field, but rather a field with the following properties: large deformations and local perturbations. The "local" field is generated from 2000 voxels which are randomly picked in the volume. For each voxel, each of the 3 components of the deformation is the realization of a Gaussian random variable of standard deviation $120 \mathrm{~mm}$. We then perform a local smoothing in order to propagate this perturbation to a local neighborhood while preserving discontinuities. We compare the multigrid method with a global affine registration method, in which a 12-parameter deformation is estimated for the entire volume.

As we have the binary classification of the phantom, we can assess the quality of the registration based on the overlap of two volumes: the first volume is the initial classification, i.e. a gold standard (grey matter/white matter), the second volume is the deformed classification, registered with the estimated deformation field. We then measure overlapping ratios like the sensibility, the specificity, and the total performance [21]. Results are presented on table 1. We also compute the mean square error (MSE) which is an indicator of the quality of the registration. Due to the use of binary classes, the resulting measures are very satisfactory. Particularly, the robustness of the method is demonstrated in critical conditions ( $9 \%$ noise and $40 \%$ inhomogeneity), which are far tougher than in any realistic acquisition.

\subsection{Experiments on a dataset of 18 sub- jects}

In order to validate the registration method on a larger database, we acquired MRI-T1 volumetric data of 18 patients. One subject was chosen as the reference subject. We then performed the registration between the reference volume (source) and each of the other subjects (target) using always the same set of parameters for the algorithm. The computation takes about 1 : 30 hour on an Ultra Sparc $30(300 \mathrm{MHz})$. The volumes are $256 \times 256 \times 200$. We use 3 levels of resolution because the displacement amplitude may reach 30 voxels.

Finally we get 17 reconstructed volumes that can be compared to the reference volume. We averaged the reconstructed volume in order to have a global overview of the quality of the method. Figure 3 presents the averaging between 17 patients after a global affine registration and the average volume after a robust multigrid registration. After global affine registration and averaging, we notice that the internal anatomical structures are blurred, because the registration is not precise enough.

However, after a robust multigrid registration, we may distinguish precisely the contours of anatomical structures, such as ventricles, deep nuclei, white matter tracks, and even cortical regions (sylvian fissure and parietal region for instance). This demonstrates the robustness of the method (robustness with respect to the acquisitions and also with respect to the algorithm parameters) over a realistic database of subjects.
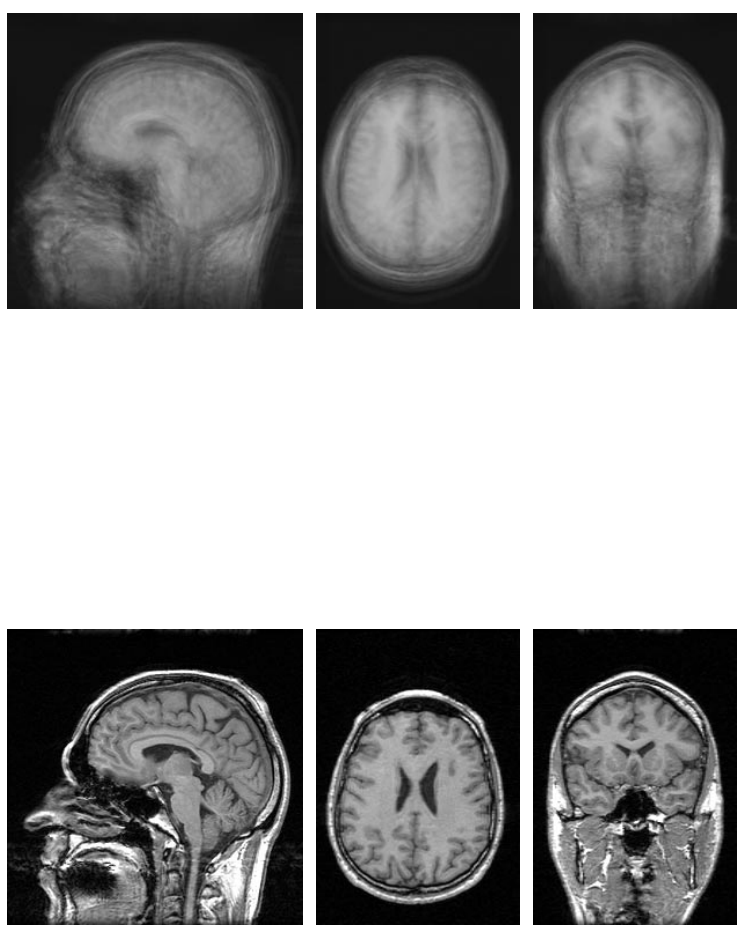

Figure 3: Results of experiments on a database of 18 subjects. Top : Averaging after a global affine registration. Middle : Averaging after a robust multigrid registration registration. Bottom: the reference subject. We keep the same set of parameters for all the subjects. This demonstrates the robustness of the method and the accuracy of the registration (after averaging we still can distinguish precisely anatomical structures such as ventricles, deep nuclei, white matter tracks and even cortical regions).

\section{Conclusion}

We have presented in this paper a new registration method based on a robust incremental $3 D$ estimation of the optical flow. We use an efficient minimization 


\begin{tabular}{|c|c|c|c|c|c|c|c|c|c|c|}
\hline & & $\begin{array}{c}\text { noise } \\
\text { inhomogeneity }\end{array}$ & $\begin{array}{l}0 \% \\
0 \%\end{array}$ & & $\begin{array}{c}\text { noise } \\
\text { inhomogeneity }\end{array}$ & $\begin{array}{l}3 \% \\
20 \%\end{array}$ & & $\begin{array}{c}\text { noise } \\
\text { inhomogeneity }\end{array}$ & $\begin{array}{c}9 \% \\
40 \%\end{array}$ & \\
\hline & & $\begin{array}{c}\text { Target } \\
\text { volume }\end{array}$ & $\begin{array}{l}\text { Grey } \\
\text { matter }\end{array}$ & $\begin{array}{l}\text { White } \\
\text { matter }\end{array}$ & $\begin{array}{c}\text { Target } \\
\text { volume }\end{array}$ & $\begin{array}{l}\text { Grey } \\
\text { matter }\end{array}$ & $\begin{array}{l}\text { White } \\
\text { matter }\end{array}$ & $\begin{array}{l}\text { Target } \\
\text { volume }\end{array}$ & $\begin{array}{c}\text { Grey } \\
\text { matter }\end{array}$ & $\begin{array}{l}\text { White } \\
\text { matter }\end{array}$ \\
\hline $\begin{array}{l}\text { Global } \\
\text { Affine }\end{array}$ & $\begin{array}{c}\text { Computation time } \\
\text { MSE } \\
\text { sensibility } \\
\text { specificity } \\
\text { total performance }\end{array}$ & $\begin{array}{c}10^{\prime} \\
964.63\end{array}$ & $\begin{array}{l}2679.49 \\
93.78 \% \\
93.16 \% \\
93.27 \%\end{array}$ & $\begin{array}{l}1751.41 \\
91.19 \% \\
93.72 \% \\
93.41 \%\end{array}$ & $\begin{array}{c}10^{\prime} \\
1104.22\end{array}$ & $\begin{array}{l}3305.50 \\
93.26 \% \\
91.69 \% \\
91.97 \%\end{array}$ & $\begin{array}{l}2171.13 \\
89.01 \% \\
92.48 \% \\
92.06 \%\end{array}$ & $\begin{array}{c}10^{\prime} \\
2005.14\end{array}$ & $\begin{array}{l}6933.05 \\
83.21 \% \\
83.19 \% \\
83.19 \%\end{array}$ & $\begin{array}{l}5031.49 \\
77.33 \% \\
85.42 \% \\
85.42 \%\end{array}$ \\
\hline $\begin{array}{c}\text { Robust } \\
\text { Multigrid }\end{array}$ & $\begin{array}{c}\text { Computation time } \\
\text { MSE } \\
\text { sensibility } \\
\text { specificity } \\
\text { total performance }\end{array}$ & $\begin{array}{c}67^{\prime} \\
138.57\end{array}$ & $\begin{array}{l}1383.46 \\
97.83 \% \\
94.28 \% \\
94.91 \%\end{array}$ & $\begin{array}{l}886.53 \\
97.35 \% \\
94.35 \% \\
94.71 \%\end{array}$ & $\begin{array}{c}85^{\prime} \\
233.23\end{array}$ & $\begin{array}{l}1534.48 \\
97.09 \% \\
94.76 \% \\
95.35 \%\end{array}$ & $\begin{array}{c}970.42 \\
96.36 \% \\
94.90 \% \\
95.03 \%\end{array}$ & $\begin{array}{c}112^{\prime} \\
667.88\end{array}$ & $\begin{array}{l}3186.49 \\
95.50 \% \\
90.73 \% \\
91.50 \%\end{array}$ & $\begin{array}{l}1463.87 \\
93.27 \% \\
93.67 \% \\
93.80 \%\end{array}$ \\
\hline
\end{tabular}

Table 1: Objective measures of the quality of the registration on simulated data. Specificity, sensibility and total performance measures are given for 3 levels of noise and 2 registration methods. We manage to recover up to 93\% of the deformation even in presence of important noise (9\%) and image intensity inhomogeneity (40\%).

framework, both multiresolution and multigrid with robust estimators. This optimization scheme is not limited to the estimation of the optical flow, but may as well be adapted to other similarity measures, leading to different registration applications. The adaptive partition of the volume accelerates the algorithm and improves the estimation in the regions of interest. Furthermore we have presented a formalism to introduce sparse local constraints in the estimation. We have objectively evaluated the benefits of this method on simulated data and demonstrated the significant impact of the method on a large database of real data.

\section{Acknowledgment}

This work has been partly supported by the Brittany Country Council under a contribution to the student grant. Grant support for the acquisition of the data was provided by the GIS Project "cognition science".

\section{References}

[1] R. Bajcsy and S. Kovacic. Multiresolution elastic matching. CVGIP, 46:1-21, 1989.

[2] M. Black and A. Rangarajan. On the unification of line processes, outlier rejection, and robust statistics with application in early vision. IJCV, 19(1):57-91, 1996.

[3] F. Bookstein. Principal warps: Thin plate splines and the decomposition of deformations. IEEE PAMI., 11(6):567585, 1989.

[4] G. Christensen. Consistent linear-elastic transformations for image matching. Proc. IPMI, pages 224-237, June 1999.

[5] D.L. Collins, A.P. Zijdenbos, V. Kollokian, J.G. Sled, N.J. Kabani, C.J. Holmes, and A.C. Evans. Design and construction of a realistic digital brain phantom. IEEE TMI, 17(3):463-468, 1998.

[6] L. Collins and A. Evans. Animal : validation and applications of nonlinear registration-based segmentation. IJPRAI, 8(11):1271-1294, 1997.
[7] L. Collins, G. Le Goualher, and A. Evans. Non linear cerebral registration with sulcal constraints. Proc. of MICCAI, pages 974-985, October 1998.

[8] W. Enkelmann. Investigations of multigrid algorithms for the estimation of optical flow fields in image sequences. CVGIP, 43(2):150-177, 1988.

[9] P. Hellier, C. Barillot, E. Mémin, and P. Pérez. Medical image registration with robust multigrid techniques. Proc. of MICCAI, pages 680-687, September 1999.

[10] B. Horn and B. Schunck. Determining optical flow. Artificial Intelligence, 17:185-203, August 1981.

[11] G. Le Goualher, C. Barillot, and Y. Bizais. Modeling cortical sulci with active ribbons. IJPRAI, 8(11):1295-1315, 1997.

[12] J. Maintz and MA. Viergever. A survey of medical image registration. MedIA, 2(1):1-36, 1998.

[13] E. Mémin and P. Pérez. A multigrid approach for hierarchical motion estimation. In Proc. ICCV, pages 933-938, January 1998.

[14] O. Musse, F. Heitz, and JP. Armspach. 3D deformable image matching using multiscale minimization of global energy functions. In Proc. CVPR, volume 2, pages $478-$ 485, June 1999

[15] M. Ono, S. Kubik, and C. Abernathey. Atlas of the cerebral sulci. Verlag, 1990.

[16] G. Subsol, JP. Thirion, and N. Ayache. A general scheme for automatically building $3 \mathrm{D}$ morphometric anatomical atlases: application to a skull atlas. MedIA, 2(1):37-60, 1998.

[17] J. Talairach and P. Tournoux. Co-planar stereotaxic atlas of the human brain. Georg Thieme Verlag, 1988.

[18] D. Terzopoulos. Image analysis using multigrid relaxation methods. IEEE PAMI, 8(2):129-139, 1986.

[19] JP. Thirion. Image matching as a diffusion process: an analogy with Maxwell's demons. MedIA, 2(3):243-260, 1998.

[20] P. Thompson and A. Toga. A surface-based technique for warping three-dimensional images of the brain. IEEE TMI 15(4):402-417, 1996.

[21] JH. Van Bemmel and MA. Musen. Handbook of medical informatics, Spinger. 